\title{
Établissement d'équations entre le diamètre et le Volume total de bois des arbres,
} adaptées au Cameroun LUC DURRIEU DE MADRON ${ }^{1}$ Bénédicte FonTEZ ${ }^{2}$ Adeline GIRAUD ${ }^{1}$ Bernard RIERA ${ }^{3}$

1 Onfi

2, avenue de Saint-Mandé 75570 Paris Cedex 12 France

2 Montpellier SupAgro - Inra Umr Mistea 2, place Pierre Viala 34060 Montpellier Cedex 2 France

3 Muséum national d'histoire naturelle - Cnrs Umr Mnhn 7179 4, avenue du Petit Château 91800 Brunoy France

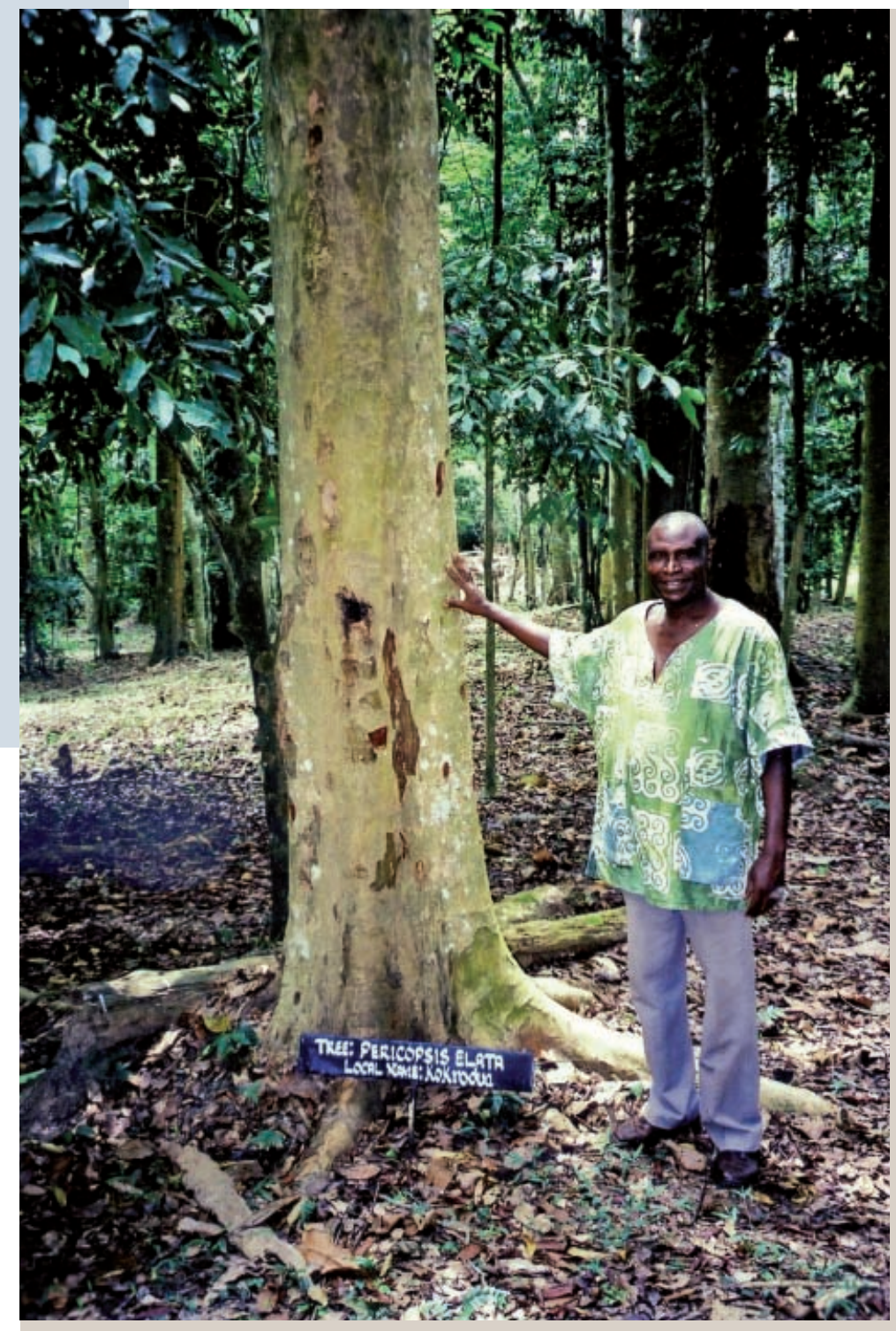

Pericopsis elata, nom commercial assamela, nom vernaculaire krokodua. Photo H. F. Maître. 
RÉSUMÉ

\section{ÉTABLISSEMENT D’ÉQUATIONS ENTRE LE DIAMÈTRE ET LE VOLUME TOTAL DE BOIS DES ARBRES, ADAPTÉES AU CAMEROUN}

Le mécanisme financier de la Réduction des émissions dues à la déforestation et à la dégradation des forêts (Redd) intéresse les pays du bassin du Congo, qui disposent de peu de données précises sur les stocks de carbone en forêt. Les principales équations allométriques pantropicales disponibles ont pour paramètres : le diamètre, la hauteur et la densité spécifique du bois; mais aucun des arbres mesurés ne provient de forêts africaines. Les rares équations établies en Afrique s'avèrent insuffisantes, notamment par déficit d'échantillon en arbres de grande taille. Ainsi, pour pallier cette carence, 55 arbres de 10 à $120 \mathrm{~cm}$ de diamètre, appartenant à 23 espèces, ont été mesurés en forêt dense semidécidue du Sud-Est du Cameroun. Après abattage, les arbres tronçonnés ont été mesurés jusqu'à la découpe d'un centimètre de diamètre. Cet échantillon a permis de relier le diamètre et le volume total de bois des arbres via des modèles polynomiaux et non linéaires (additif et multiplicatif). La biomasse des arbres pourra être alors déduite par l'utilisation de la densité spécifique et un facteur correctif pour le feuillage. Dans la perspective de cette étude, afin d'affiner les mesures, l'échantillon sera aug. menté lors d'une prochaine campagne de mesures, pour mieux estimer le volume total des gros arbres.

Mots-clés : biomasse, équation allométrique, stock de carbone, Redd, Cameroun.

\section{ABSTRACT}

\section{FORMULATING AN EQUATION FOR CAMEROON TIMBER BETWEEN TREE DIAMETER AND THE TOTAL TIMBER VOLUME OF TREES}

The REDD mechanism (Reducing Emissions from Deforestation and forest Degradation) is of interest to the Congo Basin countries, but they have few precise data on their forest carbon stocks. The parameters for the main pan-tropical allometric equations are tree height and diameter and specific timber density, but none of the trees measured are from African forests. The few equations established for Africa turn out to be insufficient, in particular because large trees are not sufficiently represented in the samples measured. To address this data deficit, we measured 55 trees with a diameter of 10 to $120 \mathrm{~cm}$, from 23 different species in a dense semi-deciduous forest in south-eastern Cameroon. After felling, the logs were cross-cut and measured until the one centimetre in diameter cutting. The sample was used to link the diameter and total timber volume of the trees via polynomial non-linear models (addition and multiplication). Then tree biomass should be deduced by applying the specific density and a correction factor for foliage. In order to refine these measurements, the sample will be enlarged during a further measurement campaign to produce a closer estimation of the total volume of huge trees.

Keywords: biomass, allometric equations, carbon stocks, REDD, Cameroon.

\section{RESUMEN}

\section{ESTABLECIMIENTO DE ECUACIONES ENTRE EL DIÁMETRO Y EL VOLUMEN TOTAL DE MADERA, ADAPTADAS A CAMERÚN}

El mecanismo financiero de la Reducción de Emisiones por la Deforestación y la Degradación (REDD) interesa a los países de la cuenca del Congo, que disponen de pocos datos precisos sobre las reservas de carbono en el bosque. Los parámetros de las principales ecuaciones pantropicales disponibles son: diámetro, altura y densidad específica de la madera; pero ninguno de los árboles medidos procedía de bosques africanos. Las pocas ecuaciones establecidas en África resultan insuficientes, principalmente por falta de muestra en árboles de gran tamaño. Así pues, para paliar esta carencia, se midieron 55 árboles de 10 a $120 \mathrm{~cm}$ de diámetro, pertenecientes a 23 especies, en el bosque tropical semicaducifoliado del sudeste de Camerún. Una vez derribados, se midieron los árboles troceados hasta llegar al corte final de un centímetro de diámetro. Este muestreo permitió relacionar diámetro y volumen total de madera de los árboles a través de modelos polinomiales y no lineales (aditivos y multiplicativos). Esto permitirá deducir la biomasa de los árboles mediante la utilización de la densidad específica y de un factor correctivo de las hojarascas. Como resultado de este estudio, y para precisar las medidas, se aumentará la muestra en una próxima campaña de mediciones con el fin de calcular mejor el volumen total de árboles de talla importante.

Palabras clave: biomasa, ecuaciones alométricas, reservas de carbono, REDD, Camerún. 


\section{Introduction}

Tous les pays du bassin du Congo, y compris le Cameroun, s'intéressent aux perspectives liées au stockage de carbone en forêt naturelle, tant du point de vue de la conservation des forêts que pour la génération de crédits carbone valorisables financièrement via le mécanisme Redd (Réduction des émissions dues à la déforestation et à la dégradation des forêts).

L'évaluation des stocks de carbone en forêt naturelle se fait sur la base de l'évolution des surfaces forestières et de données sur les stocks de carbone à l'hectare. Si la télédétection peut fournir des données cartographiques assez précises sur les surfaces forestières, en revanche, peu de données de stock par hectare sont disponibles actuellement en Afrique.

L'utilisation de données d'inventaire existantes peut constituer une alternative intéressante. Dans ce cas, la biomasse totale de l'arbre s'estime en utilisant une équation de régression appelée « équation allométrique » qui relie la biomasse épigée (Agb - Above ground biomass), exprimée en mégagrammes (Mg), avec la densité spécifique du bois, le diamètre à hauteur de poitrine (Dbh) et la hauteur. Certaines équations ne prennent en compte que le diamètre et la densité spécifique du bois comme paramètres d'entrée, pour des raisons pratiques (la mesure des hauteurs est difficile en forêt tropicale). Les données sur les diamètres sont abondantes via les inventaires d'aménagement.
Les principales équations allométriques disponibles aujourd'hui (CHAVE et al., 2005) sont basées sur 27 sites et 2410 arbres dont aucun ne provient de forêts africaines. Ces équations utilisent le Dbh, la hauteur et la densité spécifique du bois (ou seulement le diamètre et la densité spécifique) comme paramètres d'entrée. D'autres équations pantropicales existent (BRown, 1997 ; GIEC, 2006), mais avec un échantillonnage moindre et toujours non africain.

Les autres réservoirs de carbone (litière, sol et bois mort) ont reçu moins d'attention.

La seule équation existante au Cameroun est celle de IBRAHIMA et al. (2002), mise au point en prenant en compte les arbres à partir de 1 centimètre $(\mathrm{cm})$ de diamètre, avec un seul arbre de diamètre supérieur à $50 \mathrm{~cm}$ sur les 71 arbres mesurés. Mais l'extrapolation en dehors de la gamme de diamètres utilisée pour estimer les modèles allométriques n'est pas toujours possible (par exemple, en Guyane, $71 \%$ de la phytomasse est constituée par les arbres de plus de $40 \mathrm{~cm}$ de diamètre) (LESCURE et al., 1983).

Deux autres équations viennent d'être mises au point au Ghana (HENRY et al., 2010) et en République démocratique du Congo (Ebuy et al., 2011). L'étude de Henry et al. (2010) concerne 42 arbres de forêt sempervirente de 2,6 à $180 \mathrm{~cm}$ de diamètre et de hauteur allant de 1,5 à 61 mètres (m),

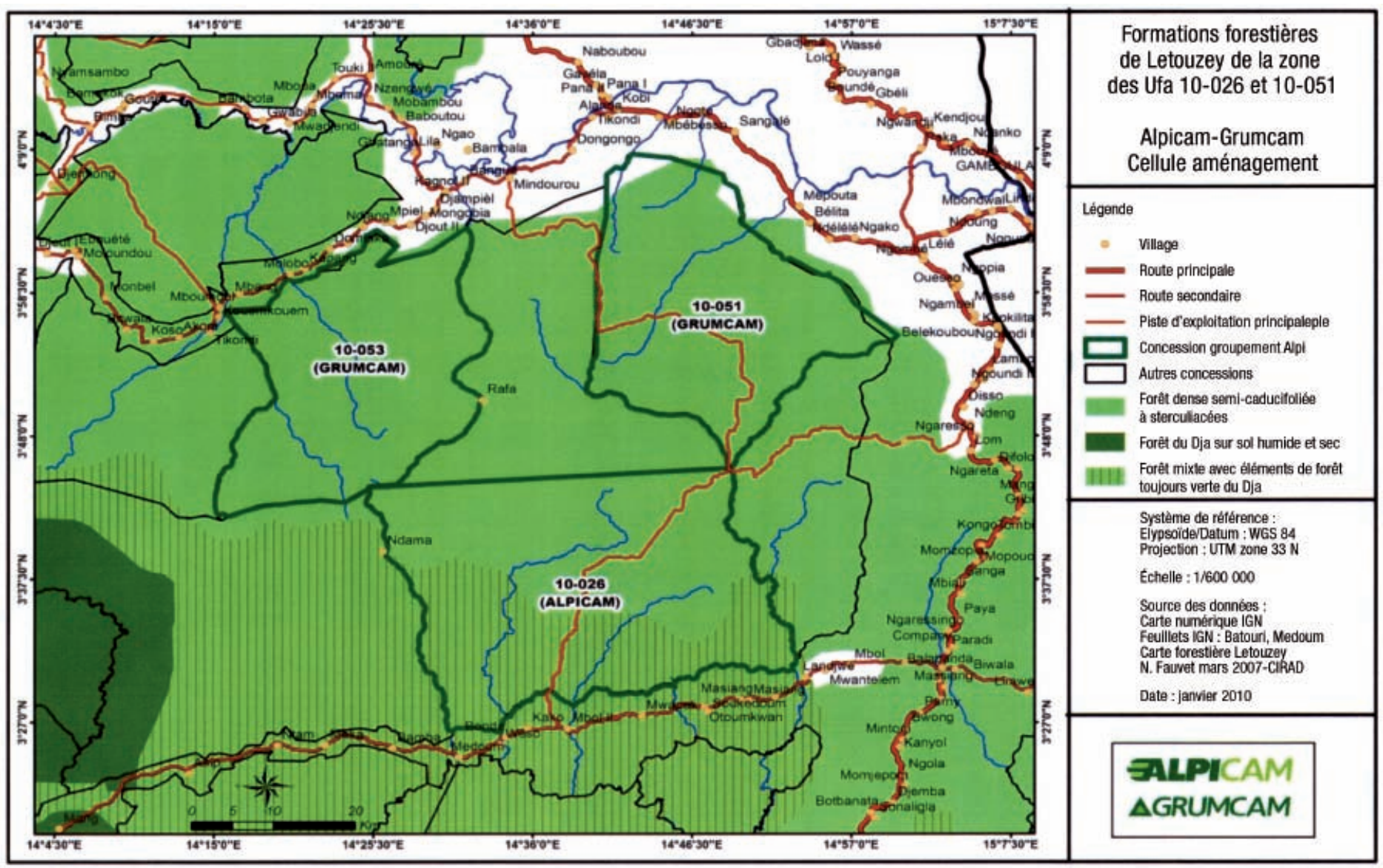

Figure 1.

Carte de végétation de Letouzey (1985) avec les deux unités

forestière d'aménagement (Ufa) où ont eu lieu les mesures d'arbres. 


\section{Échantillon}

appartenant à 16 espèces, avec une pluviosité supérieure à 2000 millimètres par an $(\mathrm{mm} / \mathrm{an})$. L'étude réalisée en République démocratique du Congo concerne 12 arbres de 24 à $52 \mathrm{~cm}$, appartenant à 3 espèces. L'étude de HENRY et al. (2010), outre qu'elle met au point des modèles locaux, compare l'efficience des modèles pantropicaux. Il s'avère que la robustesse de ceux-ci est bonne dans certains cas (moist forests) et surtout quand sont prises en compte la hauteur des arbres et la densité spécifique des arbres. Mais les biais restent très importants (de $51 \%$ à - 3\%).

Dans l'étude de EBur et al. (2011), la biomasse épigée calculée avec les équations de CHAVE et al. (2005) se situe dans l'intervalle de confiance à $95 \%$ de l'estimation de biomasse de EBuy et al. (2011), mais avec un biais entre les estimations et les valeurs réelles mesurées de 26 à $32 \%$. Il se pose donc la question de la pertinence de ces équations pantropicales lors de leur utilisation dans le bassin du Congo. Localement, l'estimation de la biomasse peut être améliorée dans une zone donnée par une étude ciblée.

La présente étude aurait donc pour objectif principal d'établir une relation entre données dendrométriques et volume de bois total jusqu'à une découpe de $1 \mathrm{~cm}$, équation qui soit propre au Cameroun et dans la sous-région, en utilisant le mélange d'essences présent sur deux sites en forêt semidécidue camerounaise. L'étude se fonde sur le paramètre le plus simple à relever et abondamment disponible : le diamètre des arbres. Cela permettra de passer à la biomasse en utilisant la densité spécifique disponible dans la littérature (BROWN, 1997 ; GIEC, 2006 ; REYES et al., 1992 ; ZANNE et al., 2009), en rajoutant une proportion de feuilles.

Ainsi, 55 arbres de 10 à $120 \mathrm{~cm}$ de diamètre, appartenant à 23 espèces, ont été mesurés en forêt dense semi-décidue du Sud-Est camerounais.

\section{Matériel et méthode}

\section{Site}

Les arbres étudiés ont été localisés dans deux unités forestières d'aménagement (Ufa) : l'Ufa 10-026 et l'Ufa 10-051 de l'entreprise Alpicam, dans le Sud-Est du Cameroun. L'Ufa 10051 a une superficie de 86096 hectares (ha) et l'Ufa 10-026 une superficie de 126988 ha.

D’après la carte phytogéographique du Cameroun, ces Ufa sont entièrement couvertes par une forêt dense humide semicaducifoliée du domaine guinéo-congolais (LETOUZEY, 1985), dans une zone de transition se rattachant au type forêt mixte semi-caducifoliée (à sterculiacées et ulmacées). Le climat de l'Ufa 10-026 est un climat équatorial continental de type guinéen classique à deux saisons des pluies, alternant avec deux saisons sèches. La pluviosité moyenne est de 1518 mm à Mindourou (base Alpicam).
Un échantillon de 55 arbres répartis en 23 essences (tableau I) a été constitué. L'essentiel de ces arbres a été mesuré sur le futur tracé de deux routes, l'équipe de mesure précédant de quelques jours le bulldozer. En outre, dans le reste de l'assiette de coupe, des arbres fraîchement abattus ou déracinés et dont le houppier était accessible ont été mesurés, en suivant les équipes d'abattage, ainsi que des arbres à terre en bord de route.

Parmi les 55 arbres mesurés, l'elemetok, Baphia sp., est le plus représenté avec $21 \%$ des arbres mesurés, suivi de l'abalé, Petersianthus marcrocarpus (16\%), de l'ayous, Triplochyton scleroxylon (9\%), et de l'otungui, Polyalthia suaveolens (5\%). Les cinq premières essences de ce classement sont les essences les plus présentes sur le site d'inventaire.

Les mesures ont été effectuées sur les individus ayant un Dbh supérieur à $10 \mathrm{~cm}$.

Un découpage en tronçons de l'arbre a été réalisé, les branches ont été sectionnées au niveau des nœuds de ramification. Une mesure de la hauteur du tronc et de la hauteur totale de l'arbre a été faite. La base du houppier est définie à la première branche.

Une série de mesures a été effectuée sur les arbres abattus. Tous les tronçons jusqu'à un diamètre de $1 \mathrm{~cm}$ ont été mesurés en diamètre et longueur.

Le volume a été calculé à l'aide de la formule suivante (formule de Smalian) :

$$
V\left(\mathrm{~cm}^{3}\right)=((\mathrm{D} 1+\mathrm{D} 2) / 2)^{2} \pi / 4 \times \mathrm{L},
$$

avec L la longueur (en cm), D1 le diamètre moyen à la base du tronçon (en cm), D2 le diamètre moyen à l'autre extrémité du tronçon (en $\mathrm{cm})$. Les diamètres D1 et D2 sont mesurés via deux mesures perpendiculaires.

\section{Méthodes de calcul pour l'établissement d'équations donnant le volume total de bois}

La sélection d'un modèle pour estimer le volume total de bois a été réalisée avec le logiciel $R$ (fonctions gls et gnls du package nlme). Les variables connues dans cette étude étaient le Dbh et la hauteur totale $(\mathrm{H})$. Une variable construite à partir des deux précédentes a aussi été prise en compte : $\mathrm{H} \times \mathrm{Dbh}^{2}$ notée $\mathrm{HDbh}^{2}$. Cette variable a en effet été identifiée comme explicative par HENRY et al. (2010). Elle est souvent associée au diamètre du houppier dans leur étude. Le meilleur modèle est sélectionné parmi les modèles linéaires (de degré 2) à partir d'une procédure de sélection descendante pas à pas. À chaque étape, le modèle présentant le plus petit Bic (Bayesian information criterion) (SCHWARTZ, 1978) est retenu. Dans le cadre de cette étude, nous avons peu d'observations par rapport au nombre de modèles candidats. De plus, la sélection, même si elle est descendante, n'est pas ordonnée. Dans ce contexte, le critère Bic, qui pénalise plus fortement le nombre de paramètres que l'Aic (Akaike information criterion), est préférable. Les modèles polynomiaux ont été appliqués par IBRAHIMA et al. (2002) avec de bons résultats. De plus, l'examen des graphiques du volume en fonction de Dbh ou $\mathrm{H}$ indique une relation plutôt de degré 2 (quadratique) ou puissance. Afin de compléter les modèles 
Tableau I.

Essences mesurées et répartition par classe de diamètre.

\begin{tabular}{lll} 
Essences & Nom local \\
\hline Baphia sp. & Elemetok \\
\hline Triplochyton scleroxylon & Ayous \\
\hline Petersianthus marcrocarpus & Abalé (Essia) \\
\hline Polyalthia suaveolens & Otungui \\
\hline Celtis zenkeri & Diana Z \\
\hline Anonidium mannii & Ebom \\
\hline Mansonia altissima & Bété \\
\hline Strombosia pustulata & Mbang mbazoa \\
\hline Canarium schweinfurthii & Aiélé \\
\hline Rinorea sp. & Ové \\
\hline Desbordesia glaucescens & Alep \\
\hline Macaranga paxii & Assas afum \\
\hline Alstonia boonei & Emien \\
\hline Pycnanthus angolensis & Ilomba \\
\hline Gambeya africana & Longhi A \\
\hline Hylodendron gabonense & Mvanda \\
\hline Blighia welwitschii & Awonog \\
\hline Tabernae montana crasa & Etoan \\
\hline Aningeria robusta & Aniégré R \\
\hline Strombosiopsis tetrandra & Edip mbazoa \\
\hline Angylocalyx zenkeri & Mbakoa bezombo \\
\hline Isolona thonneri & Owoé \\
\hline Mammea africana & Oboto \\
\hline Trichilia welwitschii & Ebegbemva Osoé \\
\hline Picralima nitida & Obéro \\
\hline Total & \\
\hline & \\
\hline
\end{tabular}

polynomiaux, une version non linéaire est aussi proposée qui permet d'estimer la puissance associée à chaque covariable (Dbh, H, HD), en adaptant les formulations proposées par HENRY et al. (2010) à ce contexte d'étude. Pour comparer la pertinence des différents modèles ajustées, les critères du Bic, du Press (Predictive residual sum of square $=$ somme des écarts de prédiction au carré) et du coefficient $R^{2}$ généralisé (model efficiency de MAYER et BUTLER, 1993) sont résumés dans un tableau synthétique (tableau II).

Dans tous les modèles présentés ci-dessous, $V_{i}$ désigne le volume total de bois jusqu'à un diamètre découpe de $1 \mathrm{~cm}$ (en $\left.\mathrm{cm}^{3}\right), \mathrm{Dbh}_{\mathrm{i}}$ désigne le diamètre à hauteur de poitrine (en $\mathrm{cm}$ ) et $\mathrm{H}_{\mathrm{i}}$ la hauteur $(\mathrm{en} \mathrm{cm})$. Les $\varepsilon_{\mathrm{i}}$ sont supposés indépendants et de loi normale $\mathrm{N}\left(0, \sigma_{\dot{i}}\right)$. La variance est hétérogène et elle a été modélisée par une fonction puissance $\sigma_{i}{ }^{2}=\sigma^{2} \mathrm{Dbh}_{\mathrm{i}}{ }^{2 \mathrm{k}}$ (HENRY et al., 2010). Les modèles utilisés sont indiqués ci-après selon le contexte de régression.

Un premier contexte concerne le cas où les variables sont prises séparément, $V=f(D b h, H)$. Trois types de modèles sont proposés.

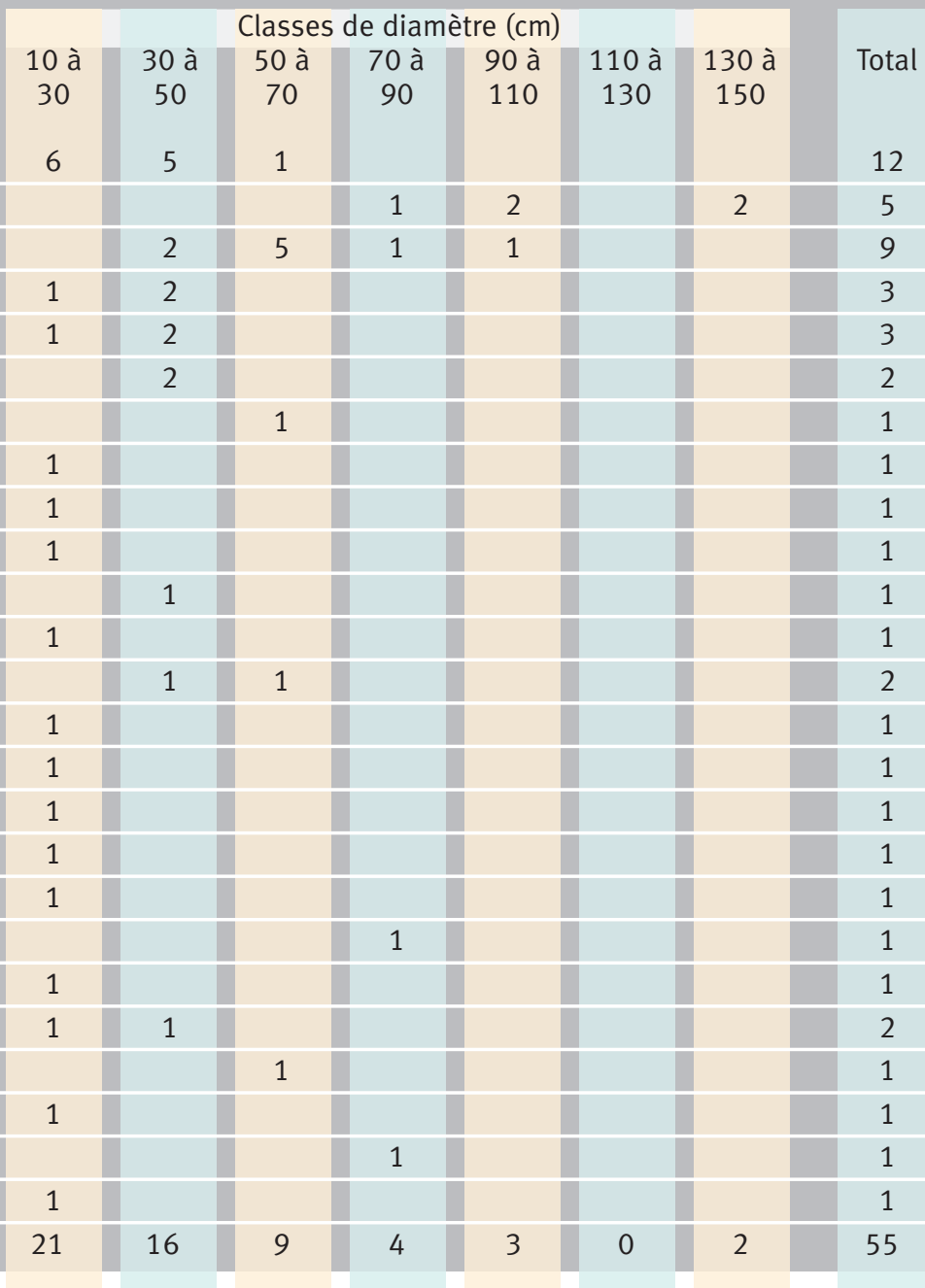

- Modèles polynomiaux

Le modèle complet est le suivant :

$V_{i}=\alpha+\beta_{D b h} D b h_{i}+\beta_{D b h 2} D b h_{i}^{2}+\beta_{H} H_{i}+\beta_{H 2} H_{i}^{2}+\varepsilon_{i}$.

Les équations intégrant la hauteur sont généralement plus fiables, puisqu'elles introduisent un paramètre de description supplémentaire (CHAVE et al., 2005). Mais les équations n'utilisant que le Dbh sont généralement plus facilement utilisables, puisque la hauteur est difficile à mesurer en forêt tropicale. Aussi, un modèle complet sans les termes $\mathrm{H}$ et $\mathrm{H}^{2} \mathrm{a}$ également été évalué.

- Modèles non linéaires (fonction puissance) : type somme

$V_{i}=\alpha+\beta_{\text {Dbh }} D_{b h}{ }^{P}+\beta_{H} H_{i}{ }^{Q}+\varepsilon_{i}$.

- Modèles non linéaires (fonction puissance) : type produit

$\mathrm{V}_{\mathrm{i}}=\alpha+\beta D b \mathrm{Dh}_{\mathrm{i}}^{\mathrm{P}} \mathrm{H}_{\mathrm{i}}^{\mathrm{Q}}+\varepsilon_{\mathrm{i}}$.

Le deuxième contexte envisagé concerne l'utilisation de la variable couplée $V=f\left(\mathrm{HbhD}^{2}\right)$ :

- Modèles polynomiaux

Le modèle complet utilisé est le suivant :

$\mathrm{V}_{\mathrm{i}}=\alpha+\beta_{\mathrm{HD}}\left(\mathrm{HbhD}^{2}{ }_{\mathrm{i}}\right)+\beta_{\mathrm{HD} 2}\left(\mathrm{HDbh}_{\mathrm{i}}{ }_{\mathrm{i}}\right)^{2}+\varepsilon_{\mathrm{i}}$. 


\section{Résultats}

\section{Sélection d'une équation pour les variables prises séparément : $\mathrm{V}=\mathrm{f}(\mathrm{Dbh}, \mathrm{H})$}

\section{Modèles polynomiaux}

Le modèle sélectionné au sens du Bic (procédure de sélection descendante) s'écrit avec l'équation (1) :

$\hat{V}=356672,6-46080,1 \mathrm{Dbh}+2300,3 \mathrm{Dbh}^{2}-31,5 \mathrm{H}$

avec $\hat{\sigma}=256,5$ et $=\hat{k} 2,16 ; \mathrm{R}^{2}=0,93$.

Intervalle de confiance à $95 \%$ pour chaque estimation de paramètre :

$\begin{array}{lcc} & 2,5 \% & 97,5 \% \\ \hat{\alpha} & 120444,2 & 592901,1 \\ \hat{\beta}_{D b h} & -67418,6 & -24741,6 \\ \hat{\beta}_{H} & -159,0 & 96,0 \\ \hat{\beta}_{D b h 2} & 1877,5 & 2722,9\end{array}$
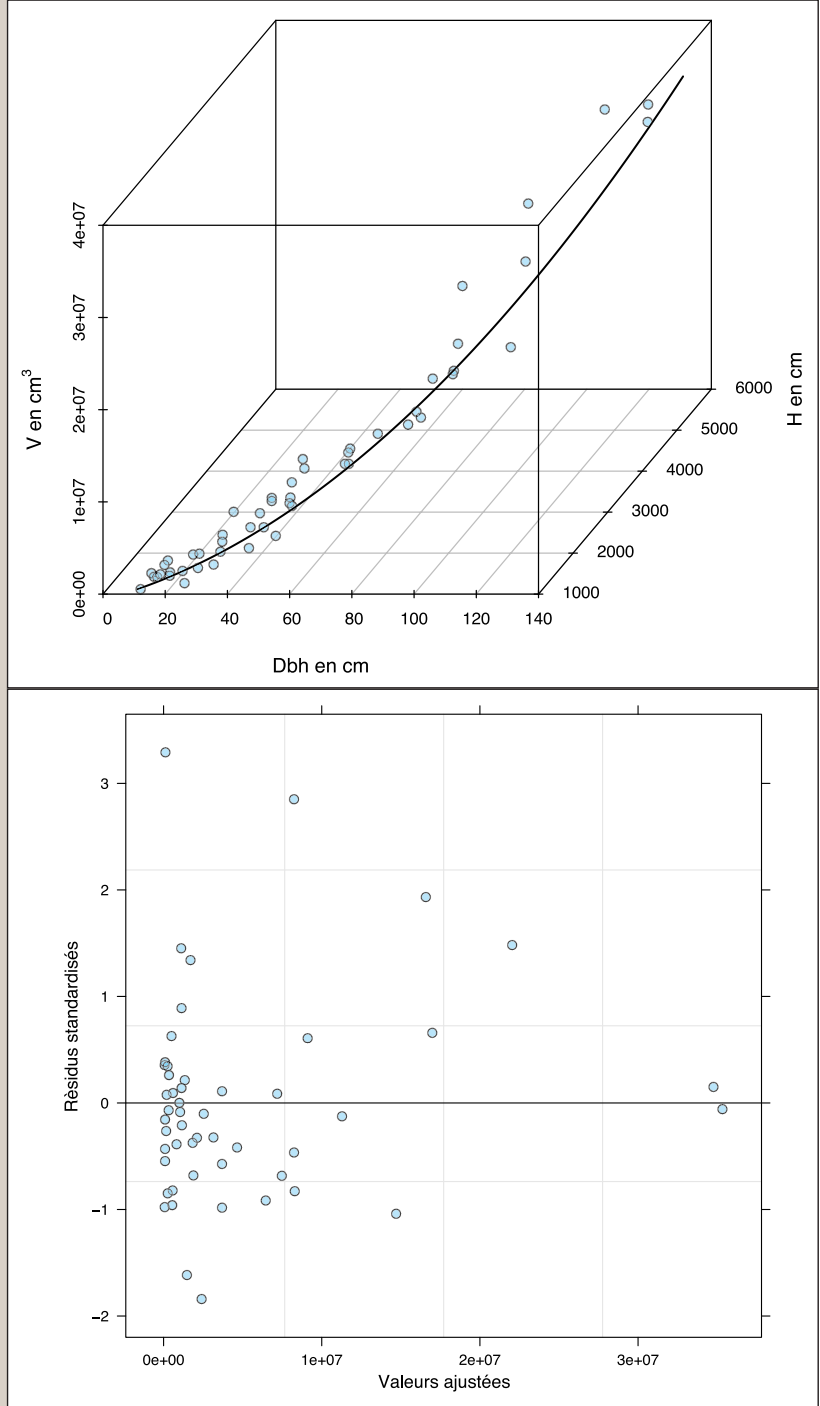

Régression polynomiale entre volume total à la découpe $1 \mathrm{~cm}$ des 55 arbres mesurés, le diamètre à hauteur de poitrine $(D b h)$ et la hauteur $(H)$.
Pour des considérations pratiques, l'équation d'ajustement sans la hauteur est aussi fournie, équation (2) : $\hat{V}=325557,3-47$ 298,4 Dbh + 2 294,2 $\mathrm{Dbh}^{2}$ avec $\hat{\sigma}=254,0$ et $=\hat{k} 2,15 ; R^{2}=0,93$.

Intervalle de confiance à $95 \%$ pour chaque estimation de paramètre :

$\begin{array}{lcc} & 2,5 \% & 97,5 \% \\ \hat{\alpha} & 127000,4 & 524114,1 \\ \hat{\beta}_{D b h} & -524114,8 & -26721,0 \\ \hat{\beta}_{D b h 2} & 1875,4 & 2713,0\end{array}$
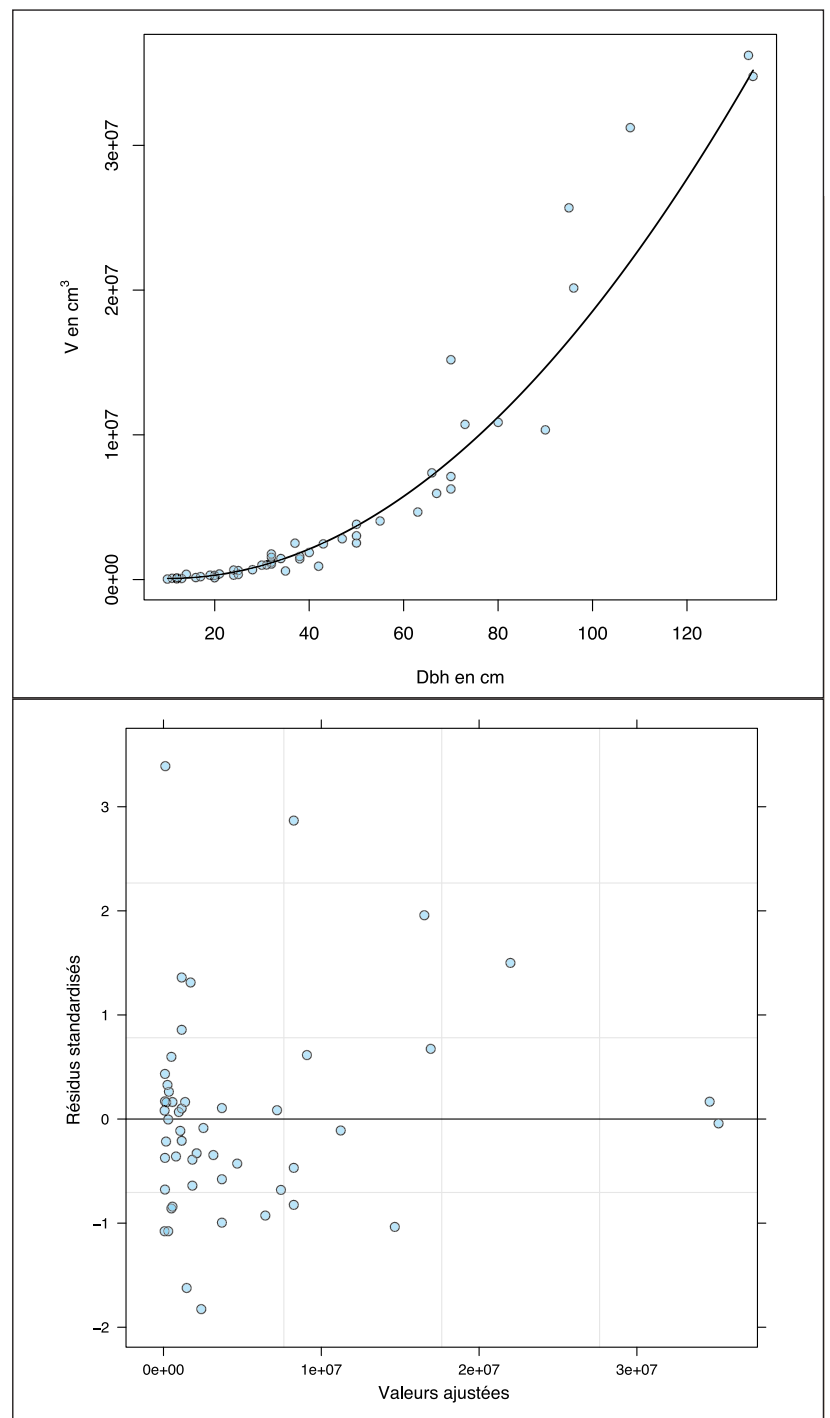

Régression polynomiale entre volume total à la découpe $1 \mathrm{~cm}$ des 55 arbres mesurés et le diamètre à hauteur de poitrine $(D b h)$. 
Modèles non linéaires (fonction puissance) : type somme

L'apport de $\mathrm{H}$ étant négligeable, un modèle plus simple (sans $\mathrm{H}$ ) est proposé. Le modèle sans ordonnée est sélectionné au sens du Bic, son estimation vaut, équation (3) :

$\hat{V}=157,9 D b h^{2,55}$

avec $\hat{\sigma}=207,8$ et $=\hat{k} 2,2 ; R^{2}=0,93$.

Intervalle de confiance à $95 \%$ pour chaque estimation de paramètre :
$\hat{\beta}_{D b h}$
$2,5 \%$
$\hat{P}$
66,7
2,4
$97,5 \%$
249,7
2,7
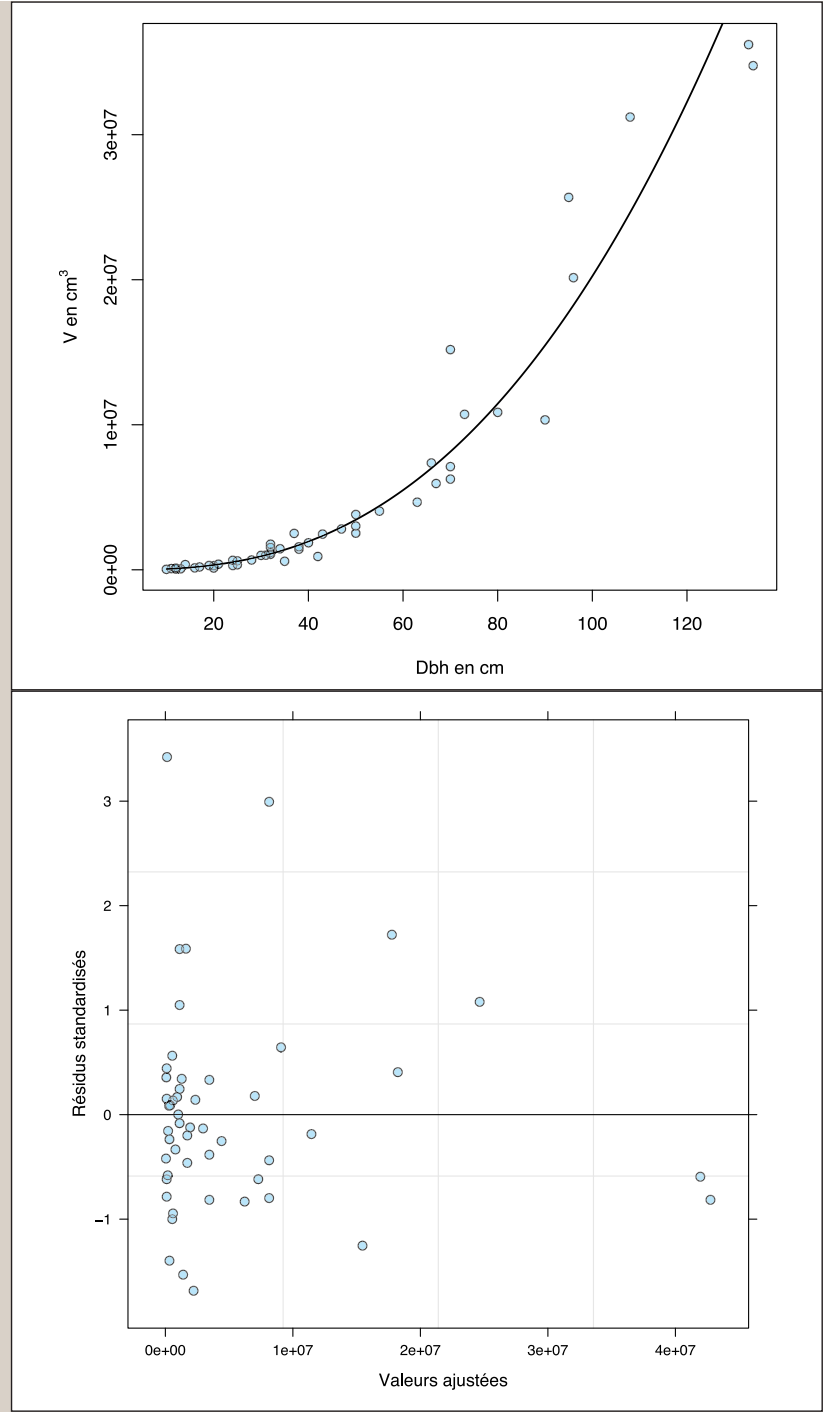

Régression non linéaire (fonction puissance) entre volume total à la découpe $1 \mathrm{~cm}$ des 55 arbres mesurés et le diamètre à hauteur de poitrine $(D b h)$.

\section{Modèles non linéaires (fonction puissance) : type produit}

Estimation du modèle, équation (4) :

$\hat{V}=1302,5+63,6 \mathrm{Dbh}^{2,5} \mathrm{H}^{0,16}$

avec $\hat{\sigma}=29,3$ et $=\hat{\kappa} 2,8 ; R^{2}=0,94$.

Intervalle de confiance à $95 \%$ pour chaque estimation de paramètre :
$2,5 \%$

$-43830,5$

$-148,1$

2,0

$-0,4$
$97,5 \%$

46435,4

275,3

2,9

0,7
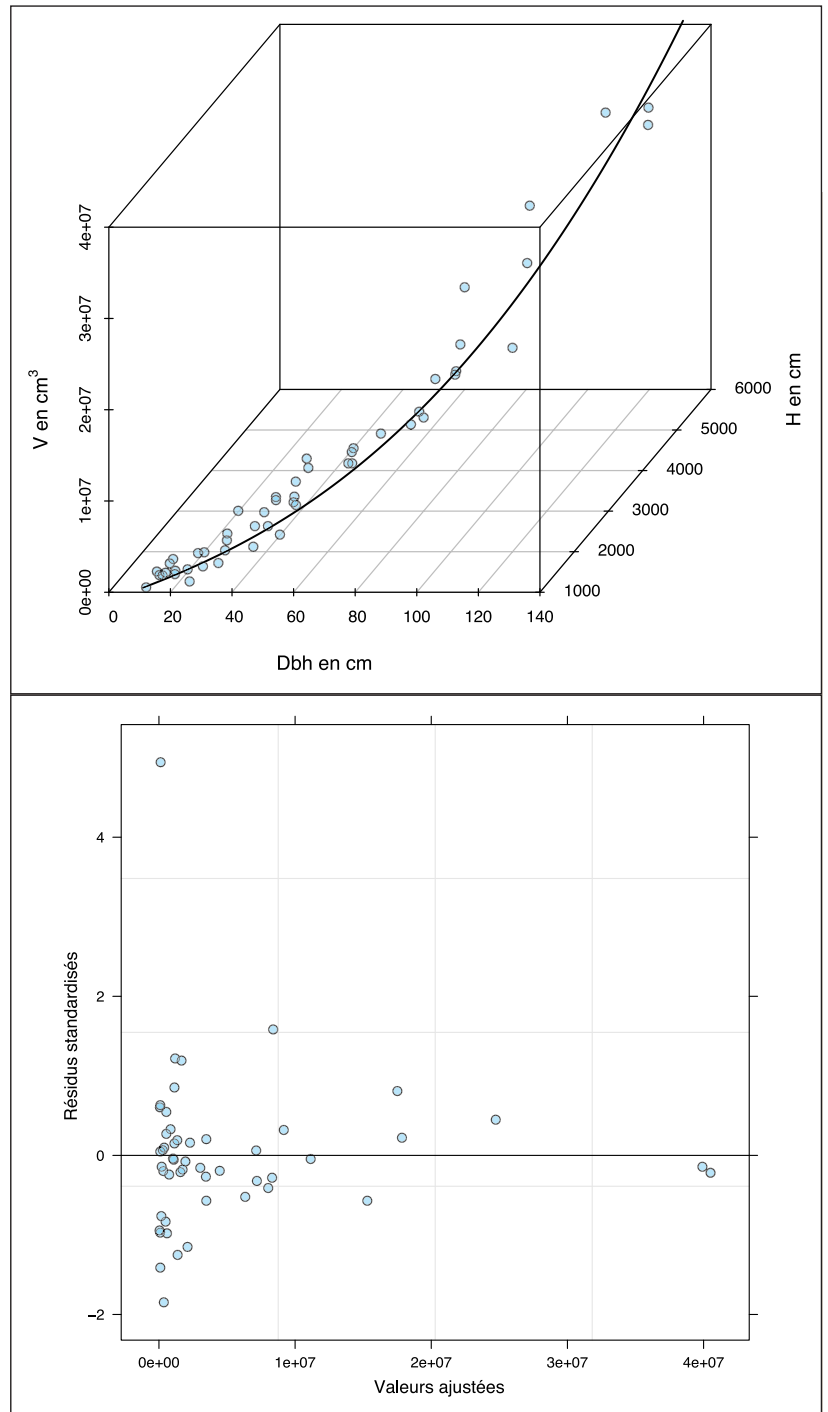

Régression non linéaire (fonction puissance type produit) entre volume total à la découpe $1 \mathrm{~cm}$ des 55 arbres mesurés, le diamètre à hauteur de poitrine $(D b h)$ et la hauteur $(H)$. 


\section{Sélection d'une équation pour la variable couplée $V=f(H D b h 2)$}

Le modèle sélectionné au sens du Bic (procédure descendante) s'écrit, équation (5) :

$\hat{V}=11$ 195,6 + 4 032,6 HDbh $^{2}$

avec $\hat{\sigma}=374,4$ et $=\hat{k} 2,02 ; \mathrm{R}^{2}=0,96$.

Intervalle de confiance à $95 \%$ pour chaque estimation de paramètre :

$\begin{array}{lcc} & 2,5 \% & 97,5 \% \\ \hat{\alpha} & -27783,4 & 50174,5 \\ \hat{\beta}_{H D b h^{2}} & 3663,7 & 4401,6\end{array}$
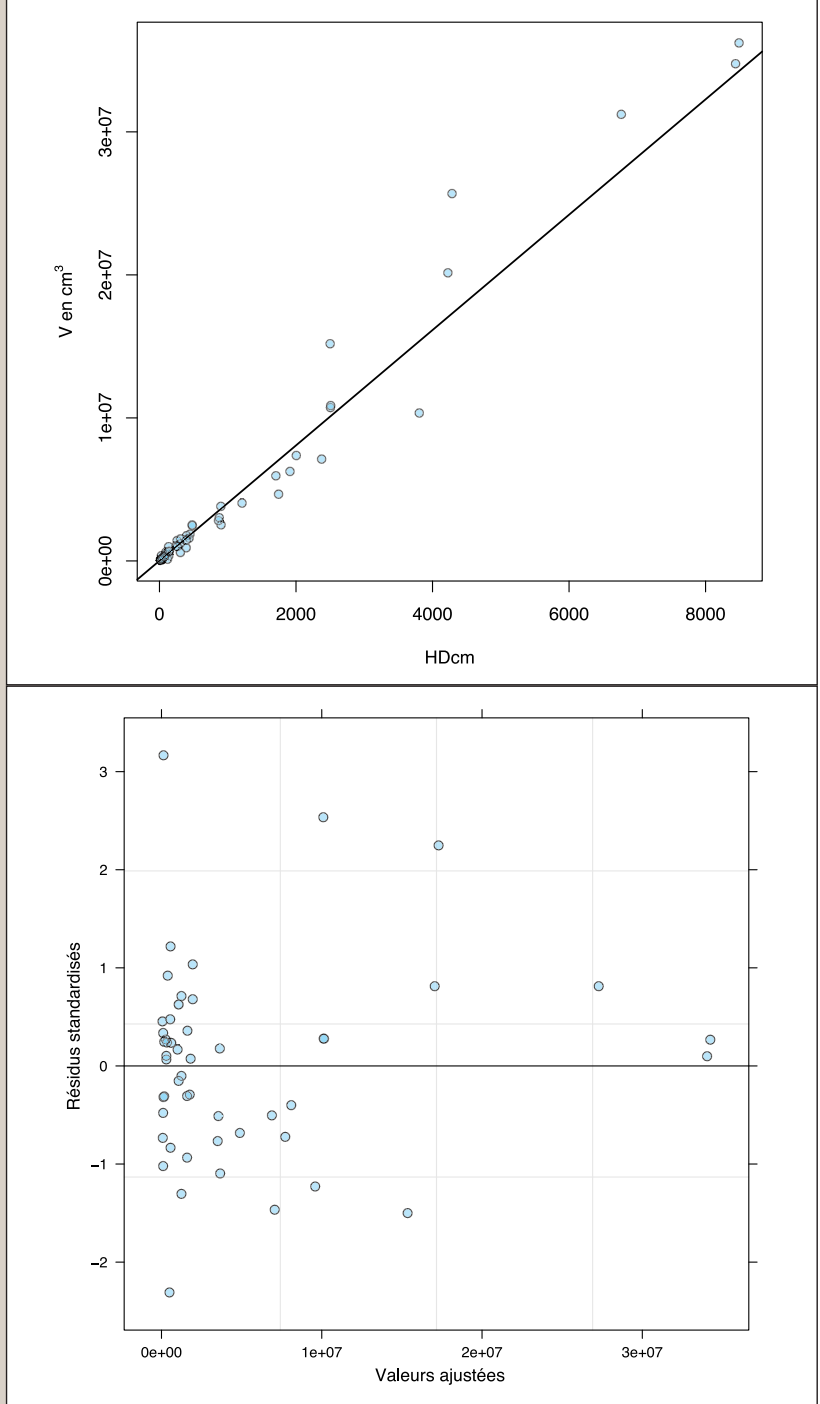

Régression non linéaire (fonction polynomiale) entre volume tota à la découpe $1 \mathrm{~cm}$ des 55 arbres mesurés, le diamètre à hauteur de poitrine $(D b h)$ et la hauteur $(H)$.
Au sens du Bic, le meilleur modèle est celui de l'équation (1), en revanche, sur les autres critères, c'est l'équation (5) la plus pertinente pour prédire le volume de bois. Notons que, sur les modèles non linéaires, c'est l'équation (4) qui a la valeur Press la plus faible. Il correspond à un produit $\mathrm{Dbh}^{2,5} \mathrm{H}^{0,16}$ en lieu et place de $\mathrm{HDbh}^{2}$. Cette puissance estimée d'environ 2,5 pour Dbh se retrouve pour tous les modèles non linéaires utilisés.

En imposant une puissance de 1 pour $\mathrm{H}$ dans l'équation (4), alors la puissance de Dbh est de 2,13 (Bic $=1$ 503,5) et sur les autres critères se retrouvent des performances équivalentes à l'équation (5) (efficacité = 0,96).

Au final, les équations (1) et (5) sont donc retenues pour estimer le volume de bois total à la découpe $1 \mathrm{~cm}$ en fonction du Dbh et de la hauteur. Si seul le Dbh est disponible, l'équation (2) est la meilleure.

La plage de validité de ces équations est de 10 à $120 \mathrm{~cm}$. Elle est valable pour les forêts semi-décidues du Cameroun, avec une pluviosité avoisinant 1500 mm/an.

\section{Passage des volumes à la biomasse}

En reprenant les données de EBuy et al., il est possible de calculer que les feuilles représentent $3 \%$ du volume du bois. De la même manière, avec les données de HENRY et al., il est obtenu 0,8 \% de feuilles. En Guyane par chez LeSCURE et al., pour les tiges de plus de $1 \mathrm{~cm}$ de diamètre, le poids sec des feuilles représentait 1,4\% du poids total sec (bois + feuilles). En Amazonie brésilienne, cette proportion est de 2,1 \% (KLINGE, RODRIGUES, 1968), et au Suriname elle est de 1,6\% (OHLER, 1980). IBRAHIMA et al. (2002) fournissent quant à eux une fraction de feuilles de 0,34\% dans la parcelle non perturbée.

Ainsi, il est possible de passer du volume à la découpe $1 \mathrm{~cm}$ à la biomasse via la densité spécifique et en ajoutant un facteur correctif pour les feuilles malgré la variabilité observée ci-dessus.

II n’y a pas de paramètre associé à la densité dans les équations présentées ci-avant ; mais un un simple calcul suffira, tel que : biomasse $=$ volume total de bois $x$ densité spécifique.

\section{Perspectives}

Les équations élaborées ici contiennent plus de 17 individus de diamètre supérieur à $45 \mathrm{~cm}$, le plus gros d'entre eux ayant $120 \mathrm{~cm}$ de diamètre. La distribution de l'échantillon traduit bien le profil du peuplement (distribution par classe de diamètre) connu par l'inventaire d'aménagement des deux concessions où a eu lieu cette étude.

Initialement, l'idée était d'avoir un échantillonnage de 10 individus par classe de diamètre ; mais les contraintes de terrain ont limité l'effectif de cette étude. Cette étude sera donc complétée en 2012 en augmentant l'échantillon, notamment pour les gros arbres qui représentent l'essentiel de la biomasse d'une forêt tropicale et qui ne sont quasiment pas représentés dans l'étude de IBRAHIMA et al. (2002).

Une pesée des feuilles sera faite pour mieux estimer cette proportion de biomasse. Les équations actuelles seront alors affinées afin de pouvoir estimer avec une bonne précision le stockage de carbone des forêts semi-décidues du Cameroun et de la sous-région.

Une comparaison sera alors possible avec les équations pantropicales existantes. 
Tableau II.

Les différents modèles testés et les différents critères permettant leur comparaison.

Équation

Équation (1)

$\hat{V}=356672,6-46080,1 \mathrm{Dbh}+2300,3 \mathrm{Dbh}^{2}-31,5 \mathrm{H}$

Équation (2)

$\hat{V}=325557,3-47$ 298,4 Dbh + 2 294,2 $\mathrm{Dbh}^{2}$

Équation (3)

$\hat{V}=157,9 \mathrm{Dbh}^{2,55}$

Équation (4)

$\hat{V}=1302,5+63,6 \mathrm{Dbh}^{2,5} \mathrm{H}^{0,16}$

Équation (5)

$\hat{V}=11$ 195,6 + 4 032,6 HDbh $^{2}$

\begin{tabular}{l|lll}
\hline R² généralisé & Rmse & Press & Bic \\
\hline 0,93 & $2,29 \mathrm{e}+6$ & $2.98 \mathrm{e}+14$ & 1454,2 \\
\hline 0,93 & $2,30 \mathrm{e}+6$ & $3.00 \mathrm{e}+14$ & 1460,9 \\
\hline 0,93 & $2,42 \mathrm{e}+6$ & $3,12 \mathrm{e}+14$ & 1505,0 \\
\hline 0,94 & $2,21 \mathrm{e}+6$ & $2,91 \mathrm{e}+14$ & 1527,0 \\
\hline 0,96 & $1,84 \mathrm{e}+6$ & $1,90 \mathrm{e}+14$ & 1467,1
\end{tabular}

Coefficient $R^{2}$ généralisé (model efficiency) de MAYER et BUTLER, 1993 ; Rmse (root-mean-square error), différence entre les valeurs prédites du modèle ou d'un estimateur et la valeur observée ; Press (Predictive residual sum of square), somme des écarts de prédiction au carré) ; Bic (Bayesian information criterion), SCHWARTZ, 1978.

\section{Remerciements}

Les auteurs remercient vivement l'entreprise Alpicam pour son aide et son accueil, notamment D. Bastin, F. Plancheron et J. Betal.

\section{Références bibliographiques}

BROWN S., LUGO A. E., 1992. Above ground biomass estimates for tropical moist forests of the Brazilian Amazon. Interciencia, 17: 8-18.

BROWN S., 1997. Estimating biomass and biomass change of tropical forests: a primer. Rome, Italie, FAO Forestry paper, 134, $55 \mathrm{p}$.

CHAVE J., ANDALO C., BROWN S., CAIRNS M. A., CHAMBERS J. Q., EAMUS D., FÖLSTER H., FROMARD F., HIGUCHI N., KIRA T., LESCURE J.-P., NELSON B. W., OGAWA H., PUIG H., RIÉRA B., YAMAKURA T., 2005. Tree allometry and improved estimation of carbon stocks and balance in tropical forests. Fcologia, 145: 87-99.

GIEC, 2006. Lignes directrices 2006 du Giec pour les inventaires nationaux de gaz à effet de serre. Vol. 4 : Agriculture, foresterie et autres affectations des terres. Kanagawa, Japon, Institute for Global Environnemental Strategies, vol. 4 : 4.46-4.52.

EBUY ALIPADE J., LOKOMBÉ DIMANDJA J. P., PONETTE Q., SONWA D., PICARD N., 2011. Biomass equation for predicting tree aboveground biomass at Yangambi, DRC. Journal of Tropical Forest Science, 23 (2): 125-132.

HENRY M., BESNARD A., ASANTE W. A., ESHUN J., ADU-BREDU S., VALENTINI R., BERNOUX M., SAINT-ANDRÉ L. 2010. Wood density, phytomass variations within and among trees, and allometric equations in a tropical rainforest of Africa. Forest Ecology and Management, 260 (8): 1375-1388.
IBRAHIMA A., SCHMIDT P., KETNER P., MOHREN G. J. M., 2002. Phytomasse et cycle des nutriments dans la forêt tropicale dense humide du sud Cameroun. Kribi, Cameroun, The Tropenbos-Cameroon Programme, Tropenbos-Cameroon Documents $9, \mathrm{XVI}+81 \mathrm{p}$.

KLINGE H., RODRIGUES W. A., 1968. Litter production in an area of Amazonian terra firme forest. Amazoniana, 1: 287302; 303-310.

LESCURE J.-P., PUIG H., RIÉRA B., LECLERC D., BEEKMAN A., BENETEAU A., 1983. La phytomasse épigée d'une forêt dense en Guyane française. Acta Fcologica, Ecologia Generalis, 4 (3) : 237-251.

LETOUZEY R., 1985. Notice de la carte phytogéographique du Cameroun au 1:500 000. Yaoundé, Cameroun, Institut de recherche agronomique, Toulouse, France, Institut de la carte internationale de la végétation, Nogent-sur-Marne, France, Cirad-Ctft, 298 p.

MAYER D. G., BUTLER D. G., 1993. Statistical validation. Ecological Modelling, 68: 21-32.

OHLER F. M. J., 1980. Phytomass and mineral content in untouched forest. Paramaribo, Suriname, University of Suriname, CELOS report, $\mathrm{n}^{\circ} 132,43 \mathrm{p}$.

PINHEIRO J. C., BATES D. M., 2000. Mixed-effects models in S and S-PLUS. New York, États-Unis, Springer-Verlag, Statistics and Computing series, $528 \mathrm{p}$.

REYES G., BROWN S., CHAPMAN J., LUGO A., 1992. Wood densities of tropical tree species. United States department of Agriculture, Forest Service, General technical reports SO-88. Southern Forest Experiment Station, 15 p.

SCHWARZ G., 1978. Estimating the Dimension of a Model. Annals of Statistics, 6: 461-464.

ZANNE A. E., LOPEZ-GONZALEZ G., COOMES D. A., ILIC J., JANSEN S., LEWIS S. L., MILLER R. B., SWENSON N. G., WIEMANN M. C., CHAVE J., 2009. Global Wood Density Database. Dryad. http://datadryad.org/handle/10255/ dryad.235. 